\title{
Second International Workshop on Verification and Evaluation of Computer and Communication Systems (VECoS 2008)
}

\author{
Leeds, UK \\ 2 - 3 July 2008
}

Editors:

Rahma Ben Ayed

Karim Djemame 


\section{Abstract}

The aim of the workshop is to bring together researchers and practitioners, in the areas of verification, control, performance, Quality of Service and dependability evaluation and assessment, to discuss the state of the art for solving the challenges facing us today in various modern computer and communication systems in which functional and non functional properties are strongly mixed.

Thus, the main motivation for VECoS is to encourage the cross-fertilisation between verification and evaluation approaches, methods and techniques especially those based on the specification formalisms for concurrent systems (i.e. process algebras, transition systems, automata networks, Petri nets, rewrite logic... etc).

This conference is sponsored by:

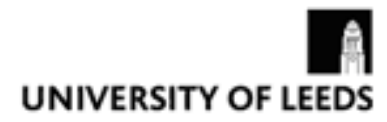




\section{Full Synopsis}

The International Workshop on Verification and Evaluation of Computer and Communication Systems (VECoS) continues its mission as one of the leading forum for the presentation of all aspects of verification, performance modelling and analysis of computer and communication systems. The first edition was held in Algiers, Algeria (2007). This year's edition is held in Leeds, United Kingdom.

VECOS offers a unique forum for researchers and practitioners from academia to share their expertise results and achievements in all areas of verification and performance evaluation of computer and communication systems including analytic modelling, simulation and measurements.

This year's programme consists of six regular paper sessions and four invited talks. Various topics are covered in the programme and include Petri nets, model checking, and wireless sensor networks.

These proceedings record the contributions from the invited speakers and from the technical sessions. We present four invited papers, nineteen technical papers, selected out of submissions from sixteen laboratories.

We have been delighted by the overwhelming response from the international community of computer and communication systems to our call for papers. The workshop attracted a large number of high quality papers from many countries. Once again we welcome back our colleagues from Tunisia, Morocco, Algeria, France, Italy, Canada, and the United Kingdom.

The programme committee includes researchers from forty laboratories. Each paper submitted was evaluated by at least three reviewers. After the evaluation, reports were returned to the programme committee for discussion and resolution of conflicts. Based on their recommendations, we implemented a consensus process, and selected the nineteen papers that we present here.

We are grateful to all members of the programme committee, the organising committee and to all referees for their hard work. The support and encouragement of the steering committee were invaluable assets. Without the support of our institutions, VECoS2008 could not have been a reality. Their recognition of the importance of this event is greatly appreciated. Finally, we would like to thank all the authors of the invited and submitted papers, and all participants at the Workshop. They are the main focus of VECoS.

We would also like to thank the School of Computing at the University of Leeds for hosting the event.

Rahma Ben Ayed and Karim Djemame

VECoS'2008 Co-Chairs

Leeds, July 2008 


\section{Editors}

This conference was edited by:

Rahma Ben Ayed - SysCom ENIT, Tunisia, Email: Rahma.BenAyed@enit.rnu.tn

Karim Djemame - University of Leeds, UK, Email: karim@comp.leeds.ac.uk

\section{Steering Committee}

Hassane Alla - LAG-INPG Grenoble, France

Nadjib Badache - LSI-USTHB Algiers, Algeria

Kamel Barkaoui (Chair) - CEDRIC-CNAM Paris, France

Rahma Ben Ayed - SysCom-ENIT Tunis, Tunisia

Karim Djouani - LISSI Universite Paris 12 Creteil, France

Mohamed Kaaniche - LAAS CNRS Toulouse, France

Patrice Moreaux - LISTIC Universite de Savoie Annecy, France

\section{Programme Committee}

Djamil Aissani - LAMOS, University of Bejaia, Algeria

Yamine Ait Ameur - ENSMA, France

Otmane Ait Mohamed - Concordia University, Canada

Nasreddine Aoumeur - University of Magdeburg, Germany

Faiza Belala - University Mentouri Constantine, Algeria

Abdelfettah Belghith - ENSI Tunis, Tunisia

Hanene Ben Abdallah - University of Sfax, Tunisia 
Narjes Ben Rajeb - LIP2 \&amp; INSAT, Tunisia

Saddek Bensalem - VERIMAG, France

Abderrahim Benslimane - University of Avignon, France

Rene Boel - Ghent University, Belgium

Hanifa Boucheneb - Ecole Polytechnique Montreal, Canada

Celine Boutrous Saab - Lamsade, Universite Paris-Dauphine, France

Maria Blanca Caminero Herraez - Universidad de Castilla-La Mancha, Spain

Allaoua Chaoui - University Mentouri Constantine, Algeria

Jean-Michel Couvreur - LIFO, Orleans University, France

Silvano Dal Zilio - LAAS CNRS Toulouse, France

Isabel Demongodin - LSIS University Paul Cezanne, France

Claude Dutheillet - LIP6 University Pierre et Marie Curie, France

Mohammed Erradi - ENSIAS, Morocco

Pascal Fontaine - LORIA University of Nancy, France

Hacene Fouchal - GRIMAAG, Universite Antilles-Guyane

Giuliana Franceschinis - Universita del Piemonte Orientale, Italy

Iain Gourlay - University of Leeds, UK

Bernd Heidergott - Vrije Universiteit Amsterdam, the Netherlands

Malika Ioualalen - USTHB Algiers, Algeria

Hanna Klaudel - IBISC CNRS, University of Evry, France

Mourad Maouche - Philadelphia University, Jordan

Herve Marchand - IRISA, France

Mohamed Mezghiche - LIFAB, University of Boumerdes, Algeria

Noufissa Mikou - University of Bourgogne, France

Bruno Monsuez - ENSTA, France

Mohamed Mosbah - LaBRI Bordeaux, France

Hassan Mountassir - LIFC University Franche-Comte Besancon, France

Laure Petrucci - LIPN CNRS, University Paris Nord, France 
Denis Poitrenaud - LIP6 - Paris VI University, France

Larbi Sekhri - University of Oran, Algeria

Tayssir Touili - LIAFA CNRS University Paris-Diderot, France

Thierry Villemur - LAAS CNRS, France

Habib Youssef - ISITCom University of Sousse, Tunisia

\section{Local Arrangements}

Mohammed Haji - University of Leeds, UK

James Padgett - University of Leeds, UK 


\section{Papers:}

\section{Session 1: Verification Methods}

Djamel Eddine Saïdouni, Nabil Belala and Messaouda Bouneb graph generation based on maximality semantics for Petri nets

Aggregation of transitions in marking http://dx.doi.org/10.14236/ewic/VECOS2008.1

Sa'ed Abed, Otmane Ait Mohamed and Ghiath Al Sammane

Multiway Decision Graphs Reduction

Approach based on the HOL Theorem Prover http://dx.doi.org/10.14236/ewic/VECOS2008.2

Jean-Michel Couvreur and Duy-Tung Nguyen Tree Data Decision Diagrams

http://dx.doi.org/10.14236/ewic/VECOS2008.3

Harry Gros-Desormeaux, Hacène Fouchal and Philippe Hunel A Comparison of Distributed Test

Generation Techniques http://dx.doi.org/10.14236/ewic/VECOS2008.4

\section{Session 2: Network Optimisation}

Abdelfettah Belghith and Wafa Akkari Handshaking Information Tapping

Power Saving Mechanisms for Ad hoc networks Based on http://dx.doi.org/10.14236/ewic/VECOS2008.5

Bouabdellah Kechar, Ahmed Louazani, Larbi Sekhri, Mohamed Faycal Khelfi Energy Efficient CrossLayer MAC Protocol for Wireless Sensor Networks ～http://dx.doi.org/10.14236/ewic/VECOS2008.6

Naouel Ben Ali, Miklos Molnar and Abdelfettah Belghith ICRA: Incremental Cycle Reduction Algorithm for optimizing multi-constrained multicast routing http://dx.doi.org/10.14236/ewic/VECOS2008.7

\section{Session 3: Network Evaluation}

Ali El Kamel and Habib Youssef REEQOS: An RSVP-TE approach for the End-to-End QoS provisioning within MPLS Domains http://dx.doi.org/10.14236/ewic/VECOS2008.8

Anis Ben Arbia and Habib Youssef Adaptive Clustering Based on Auto - Learning Algorithm http://dx.doi.org/10.14236/ewic/VECOS2008.9 


\section{Session 4: Complex Systems Analysis and Verification}

Nicolas Ayache, Loïc Correnson and Franck Védrine Verifying SystemC with Scenario http://dx.doi.org/10.14236/ewic/VECOS2008.10

Gabriella Carrozza, Marcello Cinque, Domenico Cotroneo and Roberto Natella Operating System Support to Detect Application Hangs $\quad$ http://dx.doi.org/10.14236/ewic/VECOS2008.11

Bilel Benhamamouch, Bruno Monsuez and Franck Védrine Computing WCET using symbolic execution http://dx.doi.org/10.14236/ewic/VECOS2008.12

\section{Session 5: Verification/Evaluation of Distributed Systems}

Yassine Elghayam, Mohammed Ouzzif and Mohammed Erradi An LTL Specification and Verification of a Mobile Teleconferencing System http://dx.doi.org/10.14236/ewic/VECOS2008.13

Ahmed Hammad and Hassan Mountassir Heuristics to Verify LTL Properties of Hierarchical Systems $\quad$ http://dx.doi.org/10.14236/ewic/VECOS2008.14

Boussad Addad and Said Amari Response time evaluation in Ethernet-based automation architectures http://dx.doi.org/10.14236/ewic/VECOS2008.15

\section{Session 6: Distributed Information Systems Modelling}

Shehla Abbas, Mohamed Mosbah and Akka Zemmari A Probabilistic Model for Distributed Merging of Mobile Agents http://dx.doi.org/10.14236/ewic/VECOS2008.16

Siwar Khelifi, Hatem Hadj kacem and Ahmed Hadj Kacem Specification and verification of the structural and behavioral properties of Publish/Subscribe architectures

http://dx.doi.org/10.14236/ewic/VECOS2008.17

Lu Liu, Duncan Russell, Nik Looker, David Webster and Jie Xu Evolutionary Service-Oriented 
